\title{
4-Hydroxy-2-nonenal Statues in Hypertension Patients
}

\author{
Alaa H. Jawad 1, Ammal E. Ibrahim 2, Ali Hammed 1, Zyad Al-Qaisi ${ }^{3}$, Amamer Redwan 4 \\ and Emad Yousif $1, *$ \\ ${ }^{1}$ Department of Chemistry, College of Science, Al-Nahrain University, Baghdad, Iraq \\ (alaaalqaisi74@yahoo.com; alialkarady91yahoo@gmail.com) \\ 2 Department of Pharmaceutical Chemistry, College of Pharmacy, Al-Nahrain University, Baghdad, \\ Iraq (dr.ammalalobaidi@yahoo.com) \\ ${ }^{3}$ Department of Chemistry, College of Science, University of Al-Mustansiriyah, Baghdad, Iraq \\ (zyadalqaisi@yahoo.com) \\ ${ }^{4}$ Department of Chemistry, Faculty of Science, Bani Walid University, Bani Walid, Libya \\ (amamer1982@yahoo.com) \\ * Corresponding author: emad_yousif@hotmail.com
}

\begin{abstract}
The oxidative stress is one of the main cause for cardiovascular diseases (like Hypertension) also one of the results of these diseases. This study involved 56 subjects matched ages and sex divided into two groups; 28 hypertensive subject and 28 healthy subject as control group. The following analysis was done: 4-Hydroxy-2-nonenal(4HNE) and albumin. The results show that there is a significant increase in $(4 \mathrm{HNE})$ between patients group and control group. The increase in $4 \mathrm{HNE}$ which is a product of lipid peroxidation is attributed to destruction in body cell caused by due to the increase in stress events. It has been concluded that is important nappy on ideal weight, because obesity considered main factors for heart disease and hardening of the arteries. The aim of this study was to investigate the relationship between of some oxidative stress markers and cardiovascular diseases.
\end{abstract}

Keywords: hypertension; 4-hydroxy-2-nonenal (4HNE); reactive oxygen species(ROS)

\section{Introduction}

Blood pressure is a measure of the force exercised circulation on the walls of the major arteries. hypertension is a chronic medical case which in the blood pressure in the arteries is elevated[1]. Macrophages had been supposed to be the source of the most reactive oxygen species in the vessel's wall, However, it has become clear that all the cells in the vessel wall produce ROS in different quantities and in response to diverse stimuli[2]. Cardiovascular disease is a pathological condition interrelated with cardiac valves, Oxidative stress promotes vascular smooth muscle cell proliferation and hypertrophy and collagen deposition, which leading to thickening in the vascular media and 
narrowing of the vascular cavity[3]. It has been shown that mechanical stretch to vessel wall induces ROS release. This suggests the possibility that high blood pressure itself causes raises to ROS independent of renin angiotensin system activity [3]. In addition, increased oxidative stress causes tissue damage by different mechanisms including promoting lipid peroxidation, DNA damage, and protein modification. [4]. Reactive oxygen species (ROS) are highly reactive intermediates of the oxygen metabolism, which are constantly being generated and destroyed. ROS may originate from both exogenous and endogenous sources[5]. Exogenous sources include environmental agents(like, UV or heat exposure), ionizing radiation, therapeutic agents, and tobacco smoke. Endogenous sources include mitochondria, peroxisome and inflammatory cell activation[5]. When there is an imbalance between the generation of ROS and the antioxidant defense system so that the latter becomes overwhelmed, oxidative stress occurs [2]. Oxidants and free radicals are inevitably produced during most physiological and metabolical processes, and the human body has defensive antioxidant mechanisms, these mechanisms vary according to the cell and tissue type and they may act antagonistically or synergistically[6]. Oxidative stress leads to many pathophysiological conditions in the body[7]. including neurodegenerative diseases like Parkinson's disease and Alzheimer's disease, gene mutations and cancers, chronic fatigue syndrome, heart and blood vessel Disturbance, atherosclerosis, heart, congestive heart attack and inflammatory diseases[8]. In physiological conditions, low level ROS play a role in the protection the organism, while high levels of ROS may cause damage to the structures of the cell, nucleic acids, lipids, proteins or DNA damage [9]. 4-hydroxy-2-nonenal (4HNE), a high toxicity product of lipid peroxidation, is an inhibitor of mitochondrial respiration. 4HNE It exerts its influence on respiration by inhibiting $\alpha$-ketoglutaratedehydrogenase(KGDH) [10] . a study by Teresa S.et al [11] recorded a significant increase in $4 \mathrm{HNE}$ values in hypertension patients and Juliane Cruz Campos et al 2015 observed an increase in 4HNE with hypertension and kidney disease [12]. Nitric oxide synthases (NOSs) are a family of enzymes catalyzing the production of nitric oxide (NO) from Larginine[13]. NO is an important cellular signaling molecule. Albumin represents the most abundant protein in the circulatory system with a significant antioxidant activity [14] , the antioxidant activity of albumin result from its ability to bind bilirubin, homocysteine and lipids [15] The results of the present study showed that the levels of albumin was significantly decrease $(\mathrm{p}<0.01)$ in diagnosed hypertension patients which is agree with (Oda E) in hypertension .[15]

\section{Materials and methods}

The present study comprised of 56 men divide to two groups [namely control group (28), hypertensive group (28) aged between 22-65 years. These patients were hospitalized at educational laboratories in the Al-yarmouk teaching hospital. They were divided into groups of healthy as control 
group and hypertensive patients group. Blood sample were collected and centrifuged at [4000 xg] for $5 \mathrm{~min}$ after clotting. The resultant serum were separated and stored at $[-20]{ }^{\circ} \mathrm{C}$ until used. Estimation of serum albumin was done using kit provided by BioSystems company . Serum 4-Hydroxy-2nonenal $4 \mathrm{HNE}$ is typically quantified from serum samples with the most popular method being a colorimetric assay based on biotin double antibody sandwich technology.

\section{Statistics:}

The Statistical Analysis System- SAS (2012) was used to determine of different factors in studied parameters, P-value used to significant compare between means in this study .

\section{Results and Discussion:}

Fifty six sample subjects comprising of 28 patients and 28 healthy were included in the present study, table 1 shows the means and standard deviation of age, body mass index (BMI), duration of disease , 4-Hydroxy-2-nonenal (4HNE) in addition to albumin levels for the control and patients groups.

Table 1 : Characteristics of the Hypertension (HT) and control group ( mean \pm SD) :

\begin{tabular}{|c|c|c|c|c|}
\hline \multirow{2}{*}{ P-Value } & Characteristic & Hypertension group & Control group & \\
& & $\mathrm{n}=28$ & $\mathrm{n}=28$ & \\
\hline$<0.01$ & Age (year) & $55.46 \pm 8.91$ & $23.82 \pm 5.02$ & $<0.01$ \\
\hline$<0.01$ & Body Mass index $(\mathrm{Kg} / \mathrm{m} 2)$ & $33.34 \pm 4.68$ & & \\
\hline$<0.01$ & Systolic blood pressure $(\mathrm{mmHg})$ & $14.60 \pm 1.22$ & $11.96 \pm 0.66$ & $<0.01$ \\
\hline$<0.01$ & Diastolic blood pressure $(\mathrm{mmHg})$ & $9.23 \pm 0.85$ & $8.63 \pm 0.56$ & $<0.01$ \\
\hline & & & & \\
\hline
\end{tabular}




\begin{tabular}{|c|c|c|c|c|}
\hline$<0.01$ & $4 \mathrm{HNE}(\mathrm{ng} / \mathrm{L})$ & $160.22 \pm 42.63$ & $136.46 \pm 24.55$ & $<0.01$ \\
\hline$<0.01$ & Albumin( $\mathrm{g} / \mathrm{L})$ & $4.38 \pm 0.27$ & $4.73 \pm 0.25$ & $<0.01$ \\
\hline P-Value & Characteristic & $\begin{array}{l}\text { Hypertension group } \\
\qquad n=28\end{array}$ & $\begin{array}{l}\text { Control group } \\
\qquad n=28\end{array}$ & P-Value \\
\hline
\end{tabular}

There is a significant differences $(\mathrm{p}<0.01) \quad$ in age when comparing patients groups with control group. The significant difference in BMI $(\mathrm{p}<0.01)$ between HT and control group reveal the positive correlation between obesity and disease. This finding was similar to (Ahmed A. et al2013) and (Shugar et a 12008) they found a significant increase in BMI in hypertensive patients [16,17]. Obese patients are more able to be hypertensive than lean patients, and weight gain is typically associated with increases in arterial pressure [18]. 


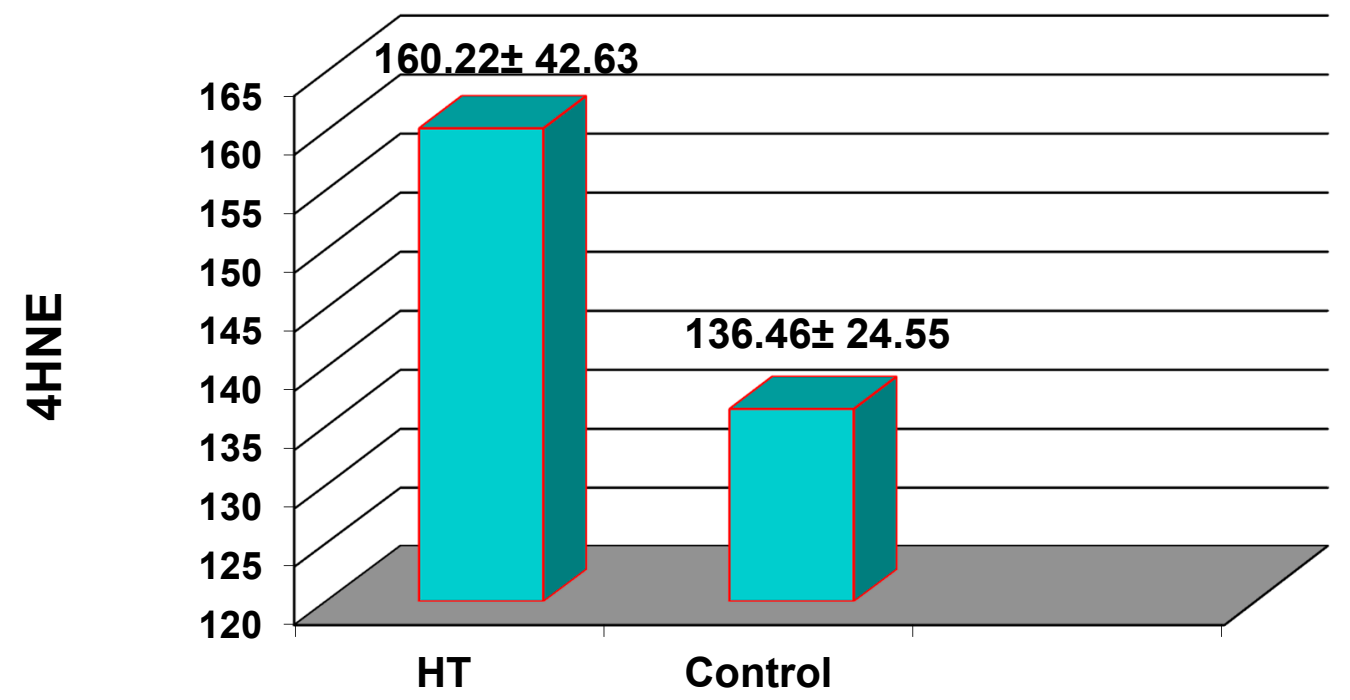

Fig.(1) Mean concentration (ng/L ) Of oxidative marker 4HNE for studied groups

The highly significant difference $(\mathrm{p}<0.01)$ of $4 \mathrm{HNE}$ in HT patient maybe due to high oxidative stress which generally causes damage to the membrane polyunsaturated fatty acids leading to the generation of 4HNE. This result agrees with study (Juliane Cruz Campos et al2015 ) [19].

Our results showed a significant difference between hypertension patient and control groups $(\mathrm{p}<0.01)$. This result was agreed with (Caroline J. Smith et al) [20]who found a significant difference in iNOS levels in hypertensive patients.

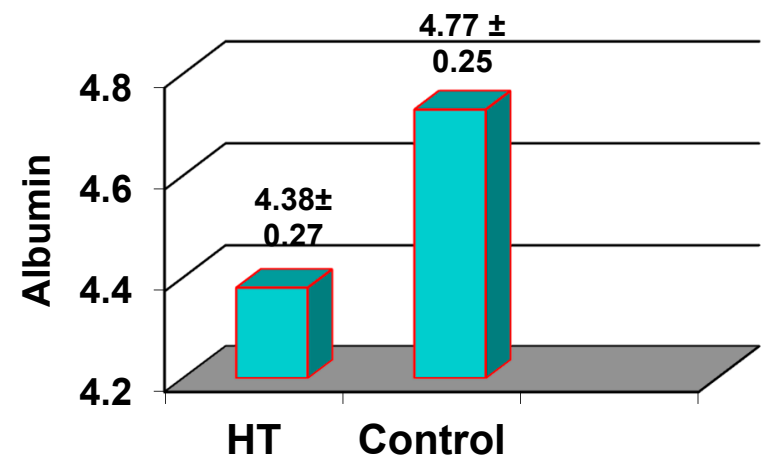

Fig.(2) Mean concentration ( $\mathrm{g} / \mathrm{L}$ ) of Albumine in the studied groups.

Albumin contains a free sulfhydryl group, this forms a disulfide with several compounds like cysteine, homocysteine, or glutathione, Albumin is able to scavenge hydroxyl radicals [21], the decrease in albumin in patient is agreed with results of (Oda E. et al2012)in Hypertension [15] who suggested that result is may be due to its function as antioxidant activity so the non-oxidized albumin is decrease in addition to negative acute phase protein, so inflammation is considered the principle cause of a decrease in the serum albumin [21]. The weak negative correlation between $4 \mathrm{HNE}$ and albumin in hypertension patient and control group 
agreed with (Giancarlo Aldini.et al2006) who found a negative correlation between $4 \mathrm{HNE}$ and Albumin.There is an evidence for a significant antioxidant activity of the represent the major and predominant circulating antioxidant in plasma known to be exposed to continuous oxidative stress.

Conclusion: Based on this study is important nappy on ideal weight, because obesity considered main factors for heart disease and hardening of the arteries.

\section{Acknowledgements}

The authors would like to thank Al-Nahrain University and Al-Yarmouk teaching hospital for the financial supports and research facilities.

\section{References:}

[1] Caroline, M. Introduction to Epidemiology: Distribution and Determinants of Disease. Cengage Learning 20136,302 . .

[2] Ahmad, A; Singhal, U.; Mohd, M.; Islam, N.; Rivisi I. The Role of the Endogenous Antioxidant Enzymes and Malondialdehyde in Essential Hypertension. Journal of Clinical and Diagnostic Research 2013,7, 987.

[3] Yasunobu H. and Hiroshi S., Hypertension and Oxidative Stress. the Journal of the Japan Medical Association.2000, 124, 1575.

[4] Dhananjay, V.; Manjusha, D.; Roshan, K.; Aasiya, S. Devendra M.and Ashlesha B. Study of Oxidative Stress in Patients with Hypertension. International J. of Recent Trends in Science And Technology 2013. 9, 157.

[5] Shiv, K. Free Radicals and Antioxidants Human and Food System. Advances in Applied Science Research $2012,2,129$.

[6] José, A. Oxidative Stress and Chronic Degenerative Disease a Role for Antioxidants. Janeza Trdine 20139 , 34.

[7] Ramalingam, M.; Kim, SJ. Reactive oxygen/nitrogen species and their functional correlations in neurodegenerative diseases. Journal of Neural Transmission 2012119 (8), 891. 
[8] Chandra, K.; Syed, A.; Abid, M.; Sweet,y R.; Najam, K. Protection Against FCA Induced Oxidative Stress Induced DNA Damage as a Model of Arthritis and In vitro Anti-arthritic Potential of Costus speciosus Rhizome Extract . International Journal of Pharmacognosy and Phytochemical Research, 2015 7(2), 383.

[9] Chandra, K.; Syed, A.; Abid, M.; Sweety, R.; Najam, K. Protection Against FCA Induced Oxidative Stress Induced DNA Damage as a Model of Arthritis and In vitro Anti-arthritic Potential of Costus speciosus Rhizome Extract. International Journal of Pharmacognosy and Phytochemical Research, 2015 7(2), 383.

[10] Kenneth, M.; Humphries, H.; Luke I. Szweda. Selective Inactivation of $\alpha$-Ketoglutarate Dehydrogenase and Pyruvate Dehydrogenase: Reaction of Lipoic Acid with 4-Hydroxy-2-nonenal., American Chemical Society 1998 37(4), 15835.

[11] Jawad, A.; Al-Qaisi, Z.; Ibrahim, A.; Hallab, Z.; Graisa, A.; Al-Amiery, A.; Yousif, E. Effect of Anti Diabetic Drugs on Lipid Profile in Patients with Type 2 Diabetes Mellitus. Preprints 2016, 2016110071 (doi: 10.20944/preprints201611.0071.v1).

[12] Mohammed, S.; Ibrahim, A.; Hasan, A.; Yousif, E. Research Investigation on The Effect of Phytosterols on Lipid Profile. Journal of Pharmaceutical and Medicinal 2016, 2(2), 60.

[13] Elremaly, W.; Mohamed, I.; Rouleau, T.; Lavoie, J. C., Adding glutathione to parenteral nutrition prevents alveolar loss in newborn guinea pig. Free Radic. Biol. Med 2015, 87, 274.

[14] Taverna, M.; Anne-Lise, M.; Jean-Paul, M; Bertrand, G. Specific antioxidant properties of human serum albumin, a Review. Annals of Intensive Care, 2013, 3, 1.

[15] Jawad, A.; Ibrahim, A.; Alsayed, R.; Hallab, Z.; Al-Qaisi, Z.; Al-Amery, A.; Yousif, E. Study the Impact of Glucose-6-phosphatase Activity in Type 2 Diabetic Patients and Non Diabetic Counterparts. Preprints 2016, 2016100137 (doi: 10.20944/preprints201610.0137.v1).

[16] Ahmad, A.; Singhal, U.; Mohd, M.; Islam, N.; Rivisi, I. The Role of the Endogenous Antioxidant Enzymes and Malondialdehyde in Essential Hypertension. J Clinical Diagnostic Research, 2013, 7, 987.

[17] Shuger, S.; Xuemei, S.; Timothy, S.; Rebecca, A.; Meriwether, S. Body Mass Index as a Predictor of Hypertension Incidence Among Initially Healthy Normotensive Women. American J of Hypertension, 2008, $21(6), 613$.

[18] Lavie, C.J.; Milani, R.V.; Ventur,a H.O. Obesity and Cardiovascular Disease Risk Factor, Paradox, and Impact of Weight Loss. J American College of Cardiology, 2009, 53, 21. 
[19] Hassan, F.; Abdul Hameed, A.; Alshanon, A.; Abdullah, B.; Zaman Huri, H.; Hairunisa, N.; Yousif, E. Antitumor Activity for Gold (III) Complex by High Content Screening Technique (HCS) and Cell Viability Assay. Asian Journal of Biochemistry 2015, 10 (6), 252.

[20] Ibrahim, A.; Fadhil, D.; Jawad, A.; Thabit, J.; Alqadery, R.; Yousif, E.; Al-Amiery, A. Ginkgo Biloba for Reducing Hyperlipideamia: Case Study. Preprints 2016, 2016110134 (doi: 10.20944/preprints201611.0134.v1).

[21] Marjolaine, R.; Philippe, R.; Nihar, R.; Evelyne, T.; Emmanuel, B. The antioxidant properties of serum albumin.Review. FEBS Letters 2008, 582, 1783.

(C) 2017 by the authors. Licensee Preprints, Basel, Switzerland. This article is an open access article distributed under the terms and conditions of the Creative Commons by Attribution (CC-BY) license (http://creativecommons.org/licenses/by/4.0/). 